ANNALES

POLONICI MATHEMATICI

$95.3(2009)$

\title{
Warped product submanifolds of Kaehler manifolds with a slant factor
}

\author{
by BAYRAM SAHIN (Malatya)
}

\begin{abstract}
Recently, we showed that there exist no warped product semi-slant submanifolds in Kaehler manifolds. On the other hand, Carriazo introduced anti-slant submanifolds as a particular class of bi-slant submanifolds. In this paper, we study such submanifolds in detail and show that they are useful to define a new kind of warped product submanifolds of Kaehler manifolds. In this direction, we obtain the existence of warped product hemi-slant (anti-slant) submanifolds with examples. We give a characterization theorem and establish an inequality for the squared norm of the second fundamental form in terms of the warping function for such submanifolds. The equality case is also considered.
\end{abstract}

1. Introduction. CR-submanifolds of Kaehler manifolds were introduced by Bejancu [1] as a generalization of totally real submanifolds and holomorphic submanifolds. Since then, many papers have appeared on these submanifolds. Recently, Chen [7] (see also [8], [9]) studied warped product CR-submanifolds and showed that there exist no warped product CRsubmanifolds of the form $M_{\perp} \times_{f} M_{T}$ such that $M_{\perp}$ is a totally real submanifold and $M_{T}$ is a holomorphic submanifold of a Kaehler manifold $\bar{M}$. Then he introduced CR-warped product submanifolds as follows: A submanifold $M$ of a Kaehler manifold $\bar{M}$ is called a CR-warped product if it is the warped product $M_{T} \times_{f} M_{\perp}$ of a holomorphic submanifold $M_{T}$ and a totally real submanifold $M_{\perp}$ of $\bar{M}$. He also established general sharp inequalities for CR-warped products in Kaehler manifolds. Motivated by Chen's papers, CR-warped product submanifolds have been studied in [3], [11], [13], [14] and $[15]$.

On the other hand, slant submanifolds of Kaehler manifolds were defined by Chen in [6] as another generalization of totally real submanifolds and holomorphic submanifolds. A slant submanifold is called proper if it is

2000 Mathematics Subject Classification: 53C40, 53C42, 53C15.

Key words and phrases: warped product, slant submanifold, semi-slant submanifold, Kaehler manifold. 
neither totally real nor holomorphic. We note that there exists no inclusion relation between proper CR-submanifolds and proper slant submanifolds. In [16], N. Papaghiuc introduced a class of submanifolds, called semi-slant submanifolds; this class includes the CR-submanifolds and slant submanifolds. In [17], we proved that there do not exist warped product semi-slant submanifolds of the forms $M_{T} \times_{f} M_{\theta}$ and $M_{\theta} \times_{f} M_{T}$, where $M_{T}$ is a holomorphic submanifold and $M_{\theta}$ is a proper slant submanifold of a Kaehler manifold $\bar{M}$. Therefore we ask the following question:

Are there any warped product submanifolds of Kaehler manifolds with a slant factor?

To answer this question in the affirmative, we consider the class of antislant submanifolds defined by Carriazo [5] and show the existence of warped product anti-slant submanifolds in Kaehler manifolds. For reasons to be explained later, we prefer to use the name hemi-slant submanifold instead of anti-slant submanifold. We observe that this new class also includes proper slant and CR-submanifolds and there exists no inclusion relation between proper semi-slant submanifolds and proper hemi-slant submanifolds.

The paper is organized as follows: In Section 2, we present the basic background needed for this paper. In Section 3, we define hemi-slant submanifolds and observe that there exists no inclusion relation between the classes of semi-slant submanifolds (in the sense of Papaghiuc) and hemi-slant submanifolds. After giving two characterization theorems for hemi-slant submanifolds, we investigate the geometry of leaves of distributions which are involved in their definition. In Section 4, we prove that there do not exist warped product submanifolds of the form $M_{\perp} \times_{f} M_{\theta}$ such that $M_{\perp}$ is a totally real submanifold and $M_{\theta}$ is a proper slant submanifold of a Kaehler manifold $\bar{M}$. In Section 5, we consider warped product submanifolds of the form $M_{\theta} \times{ }_{f} M_{\perp}$ in Kaehler manifolds, give examples and a characterization theorem. We also obtain an inequality for the squared norm of the second fundamental form in terms of the warping function for warped product hemi-slant submanifolds. The equality case is also considered. The paper contains several examples.

In this paper, we assume that every object at hand is smooth and the dimension of a Kaehler manifold always means the complex dimension, unless otherwise stated.

2. Preliminaries. Let $(\bar{M}, g)$ be a Kaehler manifold. This means [18] that $\bar{M}$ admits a tensor field $J$ of type $(1,1)$ on $\bar{M}$ such that, for all $X, Y \in$ $\Gamma(T \bar{M})$, we have

$$
J^{2}=-I, \quad g(X, Y)=g(J X, J Y), \quad\left(\bar{\nabla}_{X} J\right) Y=0,
$$


where $g$ is the Riemannian metric and $\bar{\nabla}$ is the Levi-Civita connection on $\bar{M}$.

Let $\bar{M}$ be a Kaehler manifold with complex structure $J$, and $M$ a Riemannian manifold isometrically immersed in $\bar{M}$. Then $M$ is called holomorphic (or complex) if $J\left(T_{p} M\right) \subset T_{p} M$ for every $p \in M$, where $T_{p} M$ denotes the tangent space of $M$ at the point $p$, and totally real if $J\left(T_{p} M\right) \subset T_{p} M^{\perp}$ for every $p \in M$, where $T_{p} M^{\perp}$ denotes the normal space of $M$ at $p$. Besides holomorphic and totally real submanifolds, there are three other important classes of submanifolds of a Kaehler manifold determined by the behavior of the tangent bundle of the submanifold under the action of the complex structure of the ambient manifold.

(1) The submanifold $M$ is called a CR-submanifold [1] if there exists a differentiable distribution $D: p \mapsto D_{p} \subset T_{p} M$ such that $D$ is invariant with respect to $J$ and the complementary distribution $D^{\perp}$ is anti-invariant with respect to $J$.

(2) The submanifold $M$ is called slant [6] if for each non-zero vector $X$ tangent to $M$ the angle $\theta(X)$ between $J X$ and $T_{p} M$ is a constant, i.e., it does not depend on the choice of $p \in M$ and $X \in T_{p} M$.

(3) The submanifold $M$ is called semi-slant [16] if it is endowed with two orthogonal distributions $D$ and $D^{\prime}$, where $D$ is invariant with respect to $J$ and $D^{\prime}$ is slant, i.e., the angle $\theta(X)$ between $J X$ and $D_{p}^{\prime}$ is constant for $X \in D_{p}^{\prime}$.

It is clear that holomorphic (respectively, totally real) submanifolds are CRsubmanifolds (respectively, slant submanifolds) with $D^{\perp}=\{0\}$ (resp. $\theta=0$ ) and $D=\{0\}$ (resp. $\theta=\pi / 2$ ). It is also clear that CR-submanifolds and slant submanifolds are semi-slant submanifolds with $\theta=\pi / 2$ and $D=\{0\}$, respectively.

Let $M$ be a Riemannian manifold isometrically immersed in $\bar{M}$ and denote by the same symbol $g$ the Riemannian metric induced on $M$. Let $\Gamma(T M)$ be the Lie algebra of vector fields in $M$, and $\Gamma\left(T M^{\perp}\right)$ the set of all vector fields normal to $M$; the same notation is used for smooth sections of any other vector bundle $E$. Denote by $\nabla$ the Levi-Civita connection of $M$. Then the Gauss and Weingarten formulas are

$$
\begin{aligned}
& \bar{\nabla}_{X} Y=\nabla_{X} Y+h(X, Y), \\
& \bar{\nabla}_{X} N=-A_{N} X+\nabla_{X}^{\perp} N,
\end{aligned}
$$

for any $X, Y \in \Gamma(T M)$ and any $N \in \Gamma\left(T M^{\perp}\right)$, where $\nabla^{\perp}$ is the connection in the normal bundle $T M^{\perp}, h$ is the second fundamental form of $M$, and $A_{N}$ is the Weingarten endomorphism associated with $N$. The second fundamental form $h$ and the shape operator $A$ are related by

$$
g\left(A_{N} X, Y\right)=g(h(X, Y), N) .
$$


For any $X \in \Gamma(T M)$ we write

$$
J X=T X+F X,
$$

where $T X$ is the tangential component of $J X$, and $F X$ is the normal component of $J X$. Similarly, for any vector field $N$ normal to $M$, we put

$$
J N=B N+C N,
$$

where $B N$ and $C N$ are the tangential and the normal components of $J N$, respectively.

3. Hemi-slant submanifolds. In this section, we define and study hemi-slant submanifolds in a Kaehler manifold $\bar{M}$. We obtain characterizations, examples and investigate the geometry of leaves of distributions.

Definition $3.1([5])$. Let $\bar{M}$ be a Kaehler manifold and $M$ a real submanifold of $\bar{M}$. Then we say that $M$ is a hemi-slant submanifold if there exist two orthogonal distributions $D^{\perp}$ and $D^{\theta}$ on $M$ such that

(a) $T M$ admits the orthogonal direct decomposition $T M=D^{\perp} \oplus D^{\theta}$.

(b) The distribution $D^{\perp}$ is anti-invariant, i.e., $J D^{\perp} \subset T M^{\perp}$.

(c) The distribution $D^{\theta}$ is slant with slant angle $\theta$.

In this case, we call $\theta$ the slant angle of $M$. The anti-invariant distribution $D^{\perp}$ of a hemi-slant submanifold is a slant distribution with angle $\theta=\pi / 2$. It is clear that hemi-slant submanifolds are particular cases of bi-slant submanifolds (for definition, see [5]). Moreover, it is also clear that if $\theta=0$, then a hemi-slant submanifold is a CR-submanifold. Furthermore, if we denote the dimensions of $D^{\perp}$ and $D^{\theta}$ by $m_{1}$ and $m_{2}$, respectively, then we have the following:

(a) If $m_{2}=0$, then $M$ is an anti-invariant submanifold.

(b) If $m_{1}=0$ and $\theta=0$, then $M$ is an invariant submanifold.

(c) If $m_{1}=0$ and $\theta \neq 0, \pi / 2$, then $M$ is a proper slant submanifold with slant angle $\theta$.

(d) If $\theta=\pi / 2$, then $M$ is an anti-invariant submanifold.

REMARK 3.1. We note that hemi-slant submanifolds were defined by Carriazo in [5] under the name of anti-slant submanifolds as a particular class of bi-slant submanifolds. However, the term "anti-slant" may suggest that the submanifolds have no slant part, which is not the case, as one can see from Definition 5.1 and [5].

We say that a hemi-slant submanifold is proper if $m_{1} \neq 0$ and $\theta \neq$ $0, \pi / 2$. Comparing the definitions of semi-slant submanifolds and hemi-slant submanifolds, we have the following. 
Proposition 3.1. There exists no inclusion relation between the classes of proper semi-slant submanifolds and of proper hemi-slant submanifolds of Kaehler manifolds.

EXAMPLE 3.1. Let $M$ be a submanifold of $\mathbb{R}^{6}$ given by

$$
\begin{gathered}
x_{1}=\frac{\varphi}{\sqrt{2}} \cos \zeta, \quad x_{2}=\frac{\varphi}{\sqrt{2}} \sin \zeta, \quad x_{3}=\frac{\varphi}{\sqrt{2}}, \\
x_{4}=0, \quad x_{5}=t, \quad x_{6}=t, \quad \varphi \neq 0 .
\end{gathered}
$$

It is easy to see that a local frame of $T M$ is given by

$$
\begin{aligned}
& Z_{1}=-\frac{\varphi}{\sqrt{2}} \sin \zeta \frac{\partial}{\partial x_{1}}+\frac{\varphi}{\sqrt{2}} \cos \zeta \frac{\partial}{\partial x_{2}}, \\
& Z_{2}=\frac{1}{\sqrt{2}} \cos \zeta \frac{\partial}{\partial x_{1}}+\frac{1}{\sqrt{2}} \sin \zeta \frac{\partial}{\partial x_{2}}+\frac{1}{\sqrt{2}} \frac{\partial}{\partial x_{3}}, \\
& Z_{3}=\frac{\partial}{\partial x_{5}}+\frac{\partial}{\partial x_{6}} .
\end{aligned}
$$

Then using the canonical complex structure of $\mathbb{R}^{6}$, we see that $J Z_{3}$ is orthogonal to $T M$, thus $D^{\perp}=\operatorname{span}\left\{Z_{3}\right\}$. Moreover, it easy to see that $D^{\theta}=\operatorname{span}\left\{Z_{1}, Z_{2}\right\}$ is a slant distribution with slant angle $\theta=\pi / 4$. Thus $M$ is a proper hemi-slant submanifold of $\mathbb{R}^{6}$.

EXAMPLE 3.2. Let $M$ be a submanifold of $\mathbb{R}^{8}$ given by

$$
\begin{gathered}
x_{1}=\varphi, \quad x_{2}=\theta, \quad x_{3}=k \cos \theta, \quad x_{4}=k \sin \theta, \\
x_{5}=\sin \theta_{1}, \quad x_{6}=\cos \theta_{1}, \quad x_{7}=\cos \theta_{2}, \quad x_{8}=\sin \theta_{2} .
\end{gathered}
$$

It is easy to see that a local frame of $T M$ is given by

$$
\begin{aligned}
& Z_{1}=\frac{\partial}{\partial x_{2}}-k \sin \theta \frac{\partial}{\partial x_{3}}+k \cos \theta \frac{\partial}{\partial x_{4}}, \quad Z_{2}=\frac{\partial}{\partial x_{1}}, \\
& Z_{3}=\cos \theta_{1} \frac{\partial}{\partial x_{5}}-\sin \theta_{1} \frac{\partial}{\partial x_{6}}, \quad Z_{4}=-\sin \theta_{2} \frac{\partial}{\partial x_{7}}+\cos \theta_{2} \frac{\partial}{\partial x_{8}} .
\end{aligned}
$$

Then $M$ is a proper hemi-slant submanifold of $\mathbb{R}^{8}$ such that we have $D^{\perp}=$ $\operatorname{span}\left\{Z_{3}, Z_{4}\right\}$ and $D^{\theta}=\operatorname{span}\left\{Z_{1}, Z_{2}\right\}$ with slant angle $\cos ^{-1}\left(1 / \sqrt{1+k^{2}}\right)$.

Let $M$ be a hemi-slant submanifold of a Kaehler manifold $\bar{M}$. We denote the projections on the distributions $D^{\perp}$ and $D^{\theta}$ by $P_{1}$ and $P_{2}$, respectively. Then we can write

$$
X=P_{1} X+P_{2} X
$$

for any $X \in \Gamma(T M)$. Applying $J$ to (3.1) and using (2.5) we obtain

$$
J X=J P_{1} X+T P_{2} X+F P_{2} X .
$$


Thus we have

$$
\begin{gathered}
J P_{1} X \in \Gamma\left(T M^{\perp}\right), \quad T P_{1} X=0, \\
T P_{2} X \in \Gamma\left(D^{\theta}\right), \quad F P_{2} X \in \Gamma\left(T M^{\perp}\right) .
\end{gathered}
$$

Hence

$$
T X=T P_{2} X
$$

for $X \in \Gamma(T M)$.

Lemma 3.1. Let $M$ be a hemi-slant submanifold of a Kaehler manifold $\bar{M}$. Then

$$
J\left(D^{\perp}\right) \perp F\left(D^{\theta}\right) .
$$

Proof. From (2.5) we obtain $g\left(J X, F P_{2} Y\right)=g\left(J X, J P_{2} Y-T P_{2} Y\right)$ for $X \in \Gamma\left(D^{\perp}\right)$ and $Y \in \Gamma\left(D^{\theta}\right)$, hence $g\left(J X, F P_{2} Y\right)=g(J X, J Y)$. Thus, from (2.1) we have $g\left(J X, F P_{2} Y\right)=0$ as $D^{\perp}$ and $D^{\theta}$ are orthogonal.

It is known that $M$ is a slant submanifold of $\bar{M}$ if and only if

$$
T^{2}=\lambda I
$$

for some $\lambda \in[-1,0]$ (see [6]), where $I$ denotes the identity transformation of $T M$. Moreover, if $M$ is a slant submanifold and $\theta$ is the slant angle of $M$, then $\lambda=-\cos ^{2} \theta$. Thus we obtain the following characterization theorem.

Theorem 3.1. Let $D$ be a distribution on $M$. Then $D$ is slant if and only if there exists a constant $\lambda \in[-1,0]$ such that $(T P)^{2} X=\lambda X$ for $X \in \Gamma(D)$, where $P$ denotes the orthogonal projection on $D$. Moreover, in this case $\lambda=-\cos ^{2} \theta$.

Actually this theorem was proved for the Sasakian case in [4]. We can use Theorem 3.1 to characterize hemi-slant submanifolds.

TheOREM 3.2. Let $M$ be a submanifold of a Kaehler manifold $\bar{M}$. Then $M$ is a hemi-slant submanifold if and only if there exists a constant $\lambda \in$ $[-1,0]$ and a distribution $D$ on $M$ such that

(i) $D=\left\{X \in \Gamma(T M) \mid T^{2} X=\lambda X\right\}$,

(ii) for any $X \in \Gamma(T M)$ orthogonal to $D, T X=0$.

Moreover, in this case $\lambda=-\cos ^{2} \theta$, where $\theta$ denotes the slant angle of $M$.

Proof. Let $M$ be a hemi-slant submanifold of $\bar{M}$. Then $\lambda=-\cos ^{2} \theta$ and $D=D^{\theta}$. By the definition of hemi-slant submanifold, (ii) is clear. Conversely (i) and (ii) imply $T M=D \oplus D^{\perp}$. Since $T(D) \subseteq D$, from (ii) $D^{\perp}$ is an anti-invariant distribution. Thus the proof is complete.

Now we give another characterization of hemi-slant submanifolds. 
Theorem 3.3. Let $M$ be a submanifold of a Kaehler manifold $\bar{M}$. Then $M$ is a hemi-slant submanifold if and only if there exists a constant $\kappa \in$ $[-1,0]$ and a distribution $D$ on $M$ such that

(a) $D=\{X \in \Gamma(T M) \mid B F X=\kappa X\}$,

(b) for any $X \in \Gamma(T M)$ orthogonal to $D, T X=0$.

Moreover, in this case $\kappa=-\sin ^{2} \theta$, where $\theta$ denotes the slant angle of $M$.

Proof. Applying $J$ to (2.5), we obtain

$$
-X=T^{2} X+F T X+B F X+C F X
$$

for $X \in \Gamma(T M)$. Comparing the tangential and normal components, we derive

$$
-X=T^{2} X+B F X, \quad F T X+C F X=0 .
$$

Now, if $M$ is a hemi-slant submanifold, then (b) is clear. For (a), from Theorem 3.2, we have $T^{2} X=-\cos ^{2} \theta X$ for $X \in \Gamma\left(D^{\theta}\right)$. Then the first equation of (3.8) implies $B F X=-\sin ^{2} \theta X$ for $X \in \Gamma\left(D^{\theta}\right)$. Hence, $D=D^{\theta}$. Conversely, (a) and (b) imply $T M=D \oplus D^{\perp}$. Moreover, from (b) we deduce that $D^{\perp}$ is an anti-invariant distribution. From (a) and (3.8) we have

$$
-X=T^{2} X+\kappa X
$$

for $X \in \Gamma(D)$ and $\kappa \in[-1,0]$. Hence $T^{2} X=-(1+\kappa) X$ for $X \in \Gamma(D)$. Put $-(1+\kappa)=\lambda$ so that $\lambda \in[-1,0]$. Thus our assertion comes from Theorem 3.2.

From Theorem 3.3, we have the following result.

Corollary 3.1. Let $M$ be a hemi-slant submanifold of a Kaehler manifold $\bar{M}$. Then

$$
B F Y=-\sin ^{2} \theta Y, \quad C F Y=-F T Y, \quad \forall Y \in \Gamma\left(D^{\theta}\right) .
$$

In particular, we have a new characterization for slant submanifolds of Kaehler manifolds.

Theorem 3.4. Let $M$ be a submanifold of a Kaehler manifold $\bar{M}$. Then $M$ is a slant submanifold of $\bar{M}$ if and only if there exists a constant $\kappa \in$ $[-1,0]$ such that $B F X=\kappa X$ for $X \in \Gamma(T M)$.

From Theorem 3.2 we have the following lemma:

Lemma 3.2. Let $M$ be a hemi-slant submanifold of a Kaehler manifold $\bar{M}$. Then

$$
\begin{aligned}
& g(T X, T Y)=\cos ^{2} \theta g(X, Y), \\
& g(F X, F Y)=\sin ^{2} \theta g(X, Y),
\end{aligned}
$$

for $X, Y \in \Gamma\left(D^{\theta}\right)$. 
Proof. For $X, Y \in \Gamma\left(D^{\theta}\right)$, from (2.1) we have $g(T X, T Y)=g(J X-$ $F X, T Y)$. Hence $g(T X, T Y)=-g(X, J T Y)$. Using Theorem 3.2(i), we obtain (3.9). Applying Lemma 3.1 in (3.9) we get (3.10).

In the rest of this section, we study integrability of distributions and conditions under which leaves of distributions on a hemi-slant submanifold $M$ in a Kaehler manifold $\bar{M}$ are totally geodesically immersed in $M$. First, we prove the integrability of $D^{\perp}$ on a hemi-slant submanifold $M$.

TheOREM 3.5. Let $M$ be a proper hemi-slant submanifold of a Kaehler manifold $\bar{M}$. Then the anti-invariant distribution $D^{\perp}$ is integrable.

Proof. It is known that if $\bar{M}$ is a Kaehler manifold, then $d \Omega=0$, where $d$ is exterior derivative and $\Omega$ is the fundamental 2-form defined by $\Omega(X, Y)=$ $g(X, J Y)$ for $X, Y \in \Gamma(T \bar{M})$ (see [18]). Since $\Omega$ is closed $(d \Omega=0)$, for $X \in \Gamma\left(D^{\theta}\right)$ and $Y, Z \in \Gamma\left(D^{\perp}\right)$ we get

$$
\begin{aligned}
d \Omega(T X, Y, Z)= & \frac{1}{3}\{T X \Omega(Y, Z)-Y \Omega(T X, Z)+Z \Omega(T X, Y) \\
& -\Omega([T X, Y], Z)+\Omega([T X, Z], Y)-\Omega([Y, Z], T X)\}=0 .
\end{aligned}
$$

Since $D^{\perp}$ and $D^{\theta}$ are orthogonal and $D^{\perp}$ is anti-invariant, using Theorem 3.2 and (2.5) we obtain

$$
Y g(Z, F T X)+\cos ^{2} \theta g([Y, Z], X)-g([Y, Z], F T X)=0 .
$$

Since $[Y, Z] \in \Gamma(T M)$ and $F T X \in \Gamma\left(T M^{\perp}\right)$ we derive

$$
\cos ^{2} \theta g([Y, Z], X)=0 \text {. }
$$

$M$ proper implies $\theta \neq \pi / 2$, hence $[Y, Z] \in \Gamma\left(D^{\perp}\right)$.

For the slant distribution $D^{\theta}$, we have the following.

THEOREM 3.6. Let $M$ be a proper hemi-slant submanifold of a Kaehler manifold $\bar{M}$. Then the slant distribution $D^{\theta}$ is integrable if and only if

$$
F P_{2}[X, Y]=h(X, T Y)-h(T Y, X)+\nabla_{X}^{\perp} F Y-\nabla_{Y}^{\perp} F X
$$

for $X, Y \in \Gamma\left(D^{\theta}\right)$.

Proof. Using (2.1), (2.2), (2.3), (2.5), (2.6) and taking the normal part we get

$$
J P_{1} \nabla_{X} Y=h(X, T Y)+\nabla_{X}^{\perp} F Y-F P_{2} \nabla_{X} Y-C h(X, Y)
$$

for $X, Y \in \Gamma\left(D^{\theta}\right)$. Hence

$$
J P_{1}[X, Y]=h(X, T Y)-h(T Y, X)+\nabla_{X}^{\perp} F Y-\nabla_{Y}^{\perp} F X-F P_{2}[X, Y] .
$$

This proves our assertion.

According to Theorem 3.5, every hemi-slant submanifold $M$ of a Kaehler manifold $\bar{M}$ is foliated by totally real submanifolds. So in the rest of this section, we are going to study the problem when a hemi-slant submanifold is a Riemannian product of a totally real submanifold and a slant submanifold. 
TheOREM 3.7. Let $M$ be a proper hemi-slant submanifold of a Kaehler manifold $\bar{M}$. Then the distribution $D^{\theta}$ defines a totally geodesic foliation if and only if

$$
g\left(A_{J Z} T Y, X\right)=g\left(A_{F T Y} Z, X\right)
$$

for all $X, Y \in \Gamma\left(D^{\theta}\right)$ and $Z \in \Gamma\left(D^{\perp}\right)$.

Proof. From (2.2) we have $g\left(\nabla_{X} Y, Z\right)=g\left(\bar{\nabla}_{X} Y, Z\right)$ for $X, Y \in \Gamma\left(D^{\theta}\right)$ and $Z \in \Gamma\left(D^{\perp}\right)$. Then from $(2.1)$ we get $g\left(\nabla_{X} Y, Z\right)=g\left(\bar{\nabla}_{X} J Y, J Z\right)$. Using (2.5), we obtain $g\left(\nabla_{X} Y, Z\right)=g\left(\bar{\nabla}_{X} T Y, J Z\right)+g\left(\bar{\nabla}_{X} F Y, J Z\right)$. Hence,

$$
g\left(\nabla_{X} Y, Z\right)=-g\left(T Y, \bar{\nabla}_{X} J Z\right)-g\left(F Y, \bar{\nabla}_{X} J Z\right) .
$$

Now, using (2.3) and (2.1) we obtain

$$
g\left(\nabla_{X} Y, Z\right)=g\left(T Y, A_{J Z} X\right)+g\left(J F Y, \bar{\nabla}_{X} Z\right) .
$$

Then from (2.5) we get

$$
g\left(\nabla_{X} Y, Z\right)=\left(T Y, A_{J Z} X\right)+g\left(B F Y, \nabla_{X} Z\right)+g(C F Y, h(X, Z)) .
$$

Thus from Corollary 3.1 we arrive at

$$
g\left(\nabla_{X} Y, Z\right)=\left(T Y, A_{J Z} X\right)-\sin ^{2} \theta g\left(Y, \nabla_{X} Z\right)-g(F T Y, h(X, Z)) .
$$

Then from (2.4) we get

$$
\cos ^{2} \theta g\left(\nabla_{X} Y, Z\right)=g\left(A_{J Z} T Y, X\right)-g\left(A_{F T Y} Z, X\right) .
$$

This proves the assertion of theorem.

THEOREM 3.8. Let $M$ be a proper hemi-slant submanifold of a Kaehler manifold $\bar{M}$. Then the distribution $D^{\perp}$ defines a totally geodesic foliation on $M$ if and only if

$$
g\left(A_{J W} T X, Z\right)=g\left(A_{F T X} W, Z\right)
$$

for $W, Z \in \Gamma\left(D^{\perp}\right)$ and $X \in \Gamma\left(D^{\theta}\right)$.

Proof. From (2.1), (2.2), (2.3) and (2.5) we obtain

$$
g\left(\nabla_{Z} W, X\right)=-g\left(A_{J W} Z, T X\right)+g\left(J \bar{\nabla}_{Z} W, F X\right)
$$

for $W, Z \in \Gamma\left(D^{\perp}\right)$ and $X \in \Gamma\left(D^{\theta}\right)$. Using (2.2) and (2.4) we get

$$
g\left(\nabla_{Z} W, X\right)=-g\left(A_{J W} Z, T X\right)+g\left(F P_{2} \nabla_{Z} W, F X\right)-g(h(Z, W), J F X) .
$$

Thus using (2.6), (3.10) and Corollary 3.1 we derive

$$
\begin{aligned}
g\left(\nabla_{Z} W, X\right)= & -g\left(A_{J W} Z, T X\right)+\sin ^{2} \theta g\left(P_{2} \nabla_{Z} W, X\right) \\
& +g(h(Z, W), F T X) .
\end{aligned}
$$

Hence, we arrive at

$$
\cos ^{2} \theta g\left(P_{2} \nabla_{Z} W, X\right)=-g\left(A_{J W} T X, Z\right)+g\left(A_{F T X} W, Z\right),
$$

which proves the assertion.

Thus from Theorems 3.7 and 3.8 we have the following result: 
COROLlary 3.2. Let $M$ be a hemi-slant submanifold of a Kaehler manifold $\bar{M}$. Then $M$ is a locally Riemannian product manifold $M=M_{\perp} \times M_{\theta}$ if and only if

$$
A_{J W} T X=A_{F T X} W
$$

for $X \in \Gamma\left(D^{\theta}\right)$ and $W \in \Gamma\left(D^{\perp}\right)$, where $M_{\perp}$ is a totally real submanifold and $M_{\theta}$ is a slant submanifold of $\bar{M}$.

ExAmple 3.3. Consider the hemi-slant submanifold of $\mathbb{R}^{8}$ described in Example 3.2. Then $T M^{\perp}$ is spanned by $N_{1}, N_{2}, N_{3}, N_{4}$ where

$$
\begin{array}{ll}
N_{1}=\sin \theta_{1} \frac{\partial}{\partial x_{5}}+\cos \theta_{1} \frac{\partial}{\partial x_{6}}, & N_{2}=-\cos \theta_{2} \frac{\partial}{\partial x_{7}}-\sin \theta_{2} \frac{\partial}{\partial x_{8}}, \\
N_{3}=\cos \theta \frac{\partial}{\partial x_{3}}+\sin \theta \frac{\partial}{\partial x_{4}}, & N_{4}=\sin \theta \frac{\partial}{\partial x_{2}}+\frac{1}{k} \frac{\partial}{\partial x_{3}} .
\end{array}
$$

Then it is easy to see that $D^{\theta}=\operatorname{span}\left\{Z_{1}, Z_{2}\right\}$ and $D^{\perp}=\operatorname{span}\left\{Z_{3}, Z_{4}\right\}$ are integrable. Denote the leaves of $D^{\perp}$ and $D^{\theta}$ by $M_{\perp}$ and $M_{\theta}$. Then the induced metric tensor is

$$
d s^{2}=d \varphi^{2}+\left(1+k^{2}\right) d \theta^{2}+d \theta_{1}^{2}+d \theta_{2}^{2} .
$$

Thus we have

$$
g=g_{M_{\theta}}+g_{M_{\perp}} .
$$

Consequently, $M$ is a Riemannian product manifold. On the other hand, by direct computations, we have

$$
T Z_{1}=-Z_{2}, \quad T Z_{2}=\frac{1}{1+k^{2}} Z_{1} .
$$

Therefore

$$
\begin{aligned}
& F T Z_{1}=-\frac{k^{2} \sin \theta}{\left(k^{2} \sin ^{2} \theta+1\right)} N_{4}, \\
& F T Z_{2}=-\frac{k}{1+k^{2}} N_{3}-\frac{k^{2} \cos \theta}{\left(1+k^{2} \sin ^{2} \theta\right)\left(1+k^{2}\right)} N_{4} .
\end{aligned}
$$

Hence we can deduce that the condition of Corollary 3.2 is satisfied.

4. Warped products $M_{\perp} \times_{f} M_{\theta}$ in Kaehler manifolds. Let $\left(B, g_{1}\right)$ and $\left(F, g_{2}\right)$ be two Riemannian manifolds, let $f: B \rightarrow(0, \infty)$, and let $\pi: B \times F \rightarrow B$ and $\eta: B \times F \rightarrow F$ the projection maps given by $\pi(p, q)=p$ and $\eta(p, q)=q$ for every $(p, q) \in B \times F$. The warped product ([2]) $M=B \times F$ is the manifold $B \times F$ equipped with the Riemannian structure such that

$$
g(X, Y)=g_{1}\left(\pi_{*} X, \pi_{*} Y\right)+(f \circ \pi)^{2} g_{2}\left(\eta_{*} X, \eta_{*} Y\right)
$$

for all $X, Y \in \Gamma(T M)$, where $*$ denotes the tangent map. The function $f$ is called the warping function of the warped product manifold. In particular, if the warping function is constant, then the manifold $M$ is said to be trivial. 
In the following, warped product manifold will mean non-trivial warped product manifold.

Let $X, Y$ be vector fields on $B$ and $V, W$ vector fields on $F$. Then from Lemma 7.3 of [2], we have

$$
\nabla_{X} V=\nabla_{V} X=\frac{X f}{f} V
$$

where $\nabla$ is the Levi-Civita connection on $M$.

In this section we investigate the existence of warped product submanifolds $M_{\perp} \times_{f} M_{\theta}$ of Kaehler manifolds such that $M_{\perp}$ is a totally real submanifold and $M_{\theta}$ is a proper slant submanifold of $\bar{M}$. First, we have the following:

Theorem 4.1 ([17, Theorems 3.1 and 3.2]). Let $\bar{M}$ be a Kaehler manifold. Then there exist no warped product submanifolds $M=M_{T} \times_{f} M_{\theta}$ or $M=M_{\theta} \times_{f} M_{T}$ of $\bar{M}$ such that $M_{T}$ is a holomorphic submanifold and $M_{\theta}$ is a proper slant submanifold of $\bar{M}$.

In fact, Theorem 4.1 tells us that there exist no warped product semislant submanifolds (in the sense of Papaghiuc) in Kaehler manifolds. Now, we are going to investigate warped products $M=M_{\perp} \times_{f} M_{\theta}$ of a Kaehler manifold $\bar{M}$ such that $M_{\perp}$ is a totally real submanifold and $M_{\theta}$ is a proper slant submanifold of $\bar{M}$.

TheOREM 4.2. Let $\bar{M}$ be a Kaehler manifold. Then there exist no warped product submanifolds $M=M_{\perp} \times_{f} M_{\theta}$ of $\bar{M}$ such that $M_{\perp}$ is a totally real submanifold and $M_{\theta}$ is a proper slant submanifold of $\bar{M}$.

Proof. From (2.3) and (2.1) we have $g\left(A_{J W} T X, X\right)=g\left(\bar{\nabla}_{T X} W, J X\right)$ for $X \in \Gamma\left(T M_{\theta}\right)$ and $W \in \Gamma\left(T M_{\perp}\right)$. Then from (2.2) and (2.5) we get

$$
g\left(A_{J W} T X, X\right)=g\left(\nabla_{T X} W, T X\right)+g(h(T X, W), F X) .
$$

Using (4.1) we obtain

$$
g\left(A_{J W} T X, X\right)=W(\ln f) g(T X, T X)+g(h(T X, W), F X) .
$$

Thus from (2.4) and (3.10) we have

$$
g(h(T X, X), J W)=\cos ^{2} \theta W(\ln f) g(X, X)+g(h(T X, W), F X)
$$

for $X \in \Gamma\left(T M_{\theta}\right)$ and $W \in \Gamma\left(T M_{\perp}\right)$. Replacing $X$ by $T X$ in (4.2), using (3.10) and Theorem 3.2 we arrive at

$$
g(h(X, T X), J W)=-\cos ^{2} \theta W(\ln f) g(X, X)+g(h(X, W), F T X)
$$

for $X \in \Gamma\left(T M_{\theta}\right)$ and $W \in \Gamma\left(T M_{\perp}\right)$. On the other hand, from (2.3) we have $g\left(A_{F X} W, T X\right)=-g\left(\bar{\nabla}_{W} F X, T X\right)$ for $X \in \Gamma\left(T M_{\theta}\right)$ and $W \in \Gamma\left(T M_{\perp}\right)$. Then using (2.1) and (2.5) we get

$$
g\left(A_{F X} W, T X\right)=g\left(\bar{\nabla}_{W} X, J T X\right)+g\left(\nabla_{W} T X, T X\right) .
$$


Using (2.5), (2.2) and (4.1) we obtain

$g\left(A_{F X} W, T X\right)=g\left(\nabla_{W} X, T^{2} X\right)+g(h(W, X), F T X)+W(\ln f) g(T X, T X)$.

Here, considering (3.10) and Theorem 3.2, we arrive at

$$
\begin{aligned}
g\left(A_{F X} W, T X\right)= & -\cos ^{2} \theta g\left(\nabla_{W} X, X\right)+g(h(W, X), F T X) \\
& +\cos ^{2} \theta W(\ln f) g(X, X) .
\end{aligned}
$$

Then using again (4.1) and (2.4), we derive

$$
g(h(T X, W), F X)=g(h(W, X), F T X) .
$$

Thus from (4.2), (4.3) and (4.4) we conclude

$$
2 \cos ^{2} \theta W(\ln f)=0 .
$$

Since $M_{\theta}$ is proper slant and Riemannian we obtain $W(\ln f)=0$, hence $f$ is constant, which proves our assertion.

From Theorems 4.1 and 4.2 we have the following corollary.

Corollary 4.1. Let $\bar{M}$ be a Kaehler manifold. Then there exist no warped product submanifolds $M=M_{1} \times_{f} M_{\theta}$ in $\bar{M}$ such that $M_{1}$ is a holomorphic or totally real submanifold and $M_{\theta}$ is a proper slant submanifold of $\bar{M}$.

REMARK 4.1. We note that Theorem 4.2 is a generalization of Theorem 3.1 in [7]. In that case $\theta=0$.

5. Warped products $M_{\theta} \times_{f} M_{\perp}$ in Kaehler manifolds. Theorem 4.2 shows that there do not exist warped product hemi-slant submanifolds of the form $M_{\perp} \times_{f} M_{\theta}$ in Kaehler manifolds. In this section, we consider warped product hemi-slant submanifolds of the form $M_{\theta} \times_{f} M_{\perp}$, where $M_{\theta}$ is a proper slant submanifold and $M_{\perp}$ is a totally real submanifold of $\bar{M}$. First, we are going to give an example of a warped product hemi-slant submanifold of the form $M_{\theta} \times_{f} M_{\perp}$.

EXAmple 5.1. Consider a submanifold $M$ in $\mathbb{R}^{6}$ given by the equations

$$
\begin{aligned}
& x_{1}=u_{1} \cos u_{2}, \quad x_{2}=u_{3} k \cos u_{2}, \quad x_{3}=u_{1} \sin u_{2}, \\
& x_{4}=u_{3} k \sin u_{2}, \quad x_{5}=u_{3}, \quad x_{6}=u_{1}, \quad k \neq 0,1 .
\end{aligned}
$$

Then the tangent bundle $T M$ is spanned by $Z_{1}, Z_{2}$ and $Z_{3}$ where

$$
\begin{aligned}
& Z_{1}=\cos u_{2} \frac{\partial}{\partial x_{1}}+\sin u_{2} \frac{\partial}{\partial x_{3}}+\frac{\partial}{\partial x_{6}}, \\
& Z_{2}=k \cos u_{2} \frac{\partial}{\partial x_{2}}+k \sin u_{2} \frac{\partial}{\partial x_{4}}+\frac{\partial}{\partial x_{5}}, \\
& Z_{3}=-u_{1} \sin u_{2} \frac{\partial}{\partial x_{1}}-k u_{3} \sin u_{2} \frac{\partial}{\partial x_{2}}+u_{1} \cos u_{2} \frac{\partial}{\partial x_{3}}+k u_{3} \cos u_{2} \frac{\partial}{\partial x_{4}} .
\end{aligned}
$$


Then $D^{\perp}=\operatorname{span}\left\{Z_{3}\right\}$ is an anti-invariant distribution and $D^{\theta}=\operatorname{span}\left\{Z_{1}, Z_{2}\right\}$ is a slant distribution with slant angle $\cos ^{-1}\left(1-k / \sqrt{2\left(1+k^{2}\right)}\right)$. Thus $M$ is a hemi-slant submanifold of $\mathbb{R}^{6}$. It is easy to see that $D^{\theta}$ is integrable. We denote the integral manifolds of $D^{\perp}$ and $D^{\theta}$ by $M_{\perp}$ and $M_{\theta}$, respectively. Then the metric tensor $g$ of $M$ is

$$
\begin{aligned}
g & =2 d u_{1}^{2}+\left(1+k^{2}\right) d u_{3}^{2}+\left(u_{1}^{2}+k^{2} u_{3}^{2}\right) d u_{2}^{2} \\
& =g_{M_{\theta}}+\left(u_{1}^{2}+k^{2} u_{3}^{2}\right) g_{M_{\perp}} .
\end{aligned}
$$

Thus $M$ is a warped product submanifold of $\mathbb{R}^{6}$ of the form $M_{\theta} \times{ }_{f} M_{\perp}$ with warping function $\sqrt{u_{1}^{2}+k^{2} u_{3}^{2}}$.

REMARK 5.1. It is easy to see that every CR-warped product submanifold is a warped product hemi-slant submanifold of the form $M_{\theta} \times_{f} M_{\perp}$ with slant angle $\theta=0$. Thus warped product hemi-slant submanifolds of the form $M_{\theta} \times_{f} M_{\perp}$ are a generalization of CR-warped product submanifolds of Kaehler manifolds.

From now on, we will consider warped product hemi-slant submanifolds $M=M_{\theta} \times_{f} M_{\perp}$ such that $M_{\theta}$ is a proper slant submanifold and $M_{\perp}$ is a totally real submanifold of a Kaehler manifold $\bar{M}$.

Lemma 5.1. Let $M=M_{\theta} \times_{f} M_{\perp}$ be a warped product submanifold of a Kaehler manifold $\bar{M}$. Then

$$
g(h(X, Z), J W)=g(h(Z, W), F X)-T X(\ln f) g(Z, W)
$$

for $X \in \Gamma\left(T M_{\theta}\right)$ and $Z, W \in \Gamma\left(T M_{\perp}\right)$.

Proof. From (2.3) we have $g(h(T X, Z), J W)=g\left(\bar{\nabla}_{Z} T X, J W\right)$ for $X \in$ $\Gamma\left(T M_{\theta}\right)$ and $Z \in \Gamma\left(T M_{\perp}\right)$. Thus using (2.1) and (2.5) we get

$$
g(h(T X, Z), J W)=g\left(\bar{\nabla}_{Z} J X, J W\right)-g\left(\bar{\nabla}_{Z} F X, J W\right) .
$$

Using again (2.1) and (2.2) we obtain

$$
g(h(T X, Z), J W)=g\left(\nabla_{Z} X, W\right)+g\left(\bar{\nabla}_{Z} J F X, W\right) .
$$

Thus, from (4.1) and (2.6) we arrive at

$$
g(h(T X, Z), J W)=X(\ln f) g(Z, W)+g\left(\bar{\nabla}_{Z} B F X, W\right)+g\left(\bar{\nabla}_{Z} C F X, W\right) .
$$

Then Corollary 3.1, (2.2) and (2.3) imply

$$
g(h(T X, Z), J W)=X(\ln f) g(Z, W)-\sin ^{2} \theta g\left(\nabla_{Z} X, W\right)+g\left(A_{F T X} Z, W\right) \text {. }
$$

Hence, using (4.1) and (2.4) we have

$$
g(h(T X, Z), J W)=\cos ^{2} \theta X(\ln f) g(Z, W)+g(h(Z, W), F T X) .
$$

Replacing $X$ by $T X$ and using Theorem 3.2 we conclude that

$$
g(h(X, Z), J W)=g(h(Z, W), F X)-T X(\ln f) g(Z, W) .
$$


We say that a hemi-slant submanifold is mixed geodesic if

$$
h(X, Z)=0
$$

for every $X \in \Gamma\left(D^{\theta}\right)$ and $Z \in \Gamma\left(D^{\perp}\right)$.

Next, we give a characterization of warped product hemi-slant submanifolds of the form $M_{\theta} \times_{f} M_{\perp}$. First recall that we have the following result of Hiepko [12] (cf. [10, Remark 2.1]): Let $D_{1}$ be a vector subbundle in the tangent bundle of a Riemannian manifold $M$ and $D_{2}$ be its normal bundle. Suppose that the two distributions are involutive. Denote the integral manifolds of $D_{1}$ and $D_{2}$ by $M_{1}$ and $M_{2}$, respectively. Then $M$ is locally isometric to a warped product $M_{1} \times_{f} M_{2}$ if the integral manifold $M_{1}$ is totally geodesic and the integral manifold $M_{2}$ is an extrinsic sphere, i.e., $M_{2}$ is a totally umbilical submanifold with parallel mean curvature vector.

THEOREM 5.1. Let $M$ be a mixed geodesic proper hemi-slant submanifold of a Kaehler manifold $\bar{M}$. Then $M$ is a locally warped product submanifold of the form $M_{\theta} \times_{f} M_{\perp}$ if and only if

$$
A_{J Z} Y=0 \quad \text { and } \quad A_{F T Y} Z=-\cos ^{2} \theta Y(\mu) Z, \quad \forall Y \in \Gamma\left(D^{\theta}\right), Z \in \Gamma\left(D^{\perp}\right),
$$

where $\mu$ is a function on $M$ such that $W(\mu)=0$ for every $W \in \Gamma\left(D^{\perp}\right)$.

Proof. Let $M=M_{\theta} \times{ }_{f} M_{\perp}$ be a warped product submanifold of $\bar{M}$. Then $M_{\theta}$ is totally geodesic in $M$. Thus $\nabla_{X} Y \in \Gamma\left(T M_{\theta}\right)$. Then from Theorem 3.7 we have

$$
g\left(A_{J Z} T Y, X\right)=g\left(A_{F T Y} Z, X\right) .
$$

Since $M$ is mixed geodesic, using (2.4) we get $g\left(A_{J Z} T Y, X\right)=0$, which shows that $A_{J Z} T Y$ has no components in $T M_{\theta}$. On the other hand, we get

$$
g\left(A_{J Z} T Y, W\right)=g(h(T Y, W), J Z)=0
$$

since $M$ is mixed geodesic. Thus $A_{J Z} T Y=0$. Replacing $Y$ by $T Y$, and using Theorem 3.2, we have $A_{J Z} Y=0$. Then using (5.1) we obtain

$$
g(h(W, Z), F T Y)=-\cos ^{2} \theta Y(\ln f) g(Z, W) .
$$

Since $M$ mixed geodesic implies $A_{F T Y} W \in \Gamma\left(D^{\perp}\right)$, we conclude that $\mu=$ $\ln f$. Let us prove the converse. Suppose that $M$ is a mixed geodesic hemislant submanifold such that

$$
A_{J Z} Y=0 \quad \text { and } \quad A_{F T Y} Z=-\cos ^{2} \theta Y(\mu) Z, \quad \forall Y \in \Gamma\left(D^{\theta}\right), Z \in \Gamma\left(D^{\perp}\right) .
$$

Then from Theorem 3.7, $D^{\theta}$ is integrable and its integral manifold is totally geodesic in $M$. Also, from Theorem $3.5, D^{\perp}$ is always integrable in $M$. Let $M_{\perp}$ and $M_{\theta}$ be the integral manifolds of $D^{\perp}$ and $D^{\theta}$, respectively. We denote the second fundamental form of $M_{\perp}$ in $M$ by $h_{2}$. Then from (2.2) we get $g\left(h_{2}(Z, W), X\right)=g\left(\bar{\nabla}_{Z} W, X\right)$ for $Z, W \in \Gamma\left(D^{\perp}\right)$ and $X \in \Gamma\left(D^{\theta}\right)$. Using (2.1) and (2.5) we obtain $g\left(h_{2}(Z, W), X\right)=g\left(\bar{\nabla}_{Z} J W, T X\right)+g\left(\bar{\nabla}_{Z} J W, F X\right)$. 
Then taking into account that $A$ is self-adjoint, (2.1) and (2.3) imply

$$
g\left(h_{2}(Z, W), X\right)=-g\left(A_{J W} T X, Z\right)-g\left(\bar{\nabla}_{Z} W, J F X\right) .
$$

By assumption, we have $A_{J W} T X=0$. Thus using (2.6) and (2.2) we obtain

$$
g\left(h_{2}(Z, W), X\right)=-g\left(\nabla_{Z} W, B F X\right)-g(h(Z, W), C F X) .
$$

Here, considering Corollary 3.1 we have

$$
g\left(h_{2}(Z, W), X\right)=\sin ^{2} \theta g\left(\nabla_{Z} W, X\right)+g(h(Z, W), F T X) .
$$

Hence, we arrive at

$$
g\left(h_{2}(Z, W), X\right)=\sin ^{2} \theta g\left(h_{2}(Z, W), X\right)+g(h(Z, W), F T X) .
$$

As a result,

$$
g\left(h_{2}(Z, W), X\right)=\sec ^{2} \theta g\left(A_{F T X} Z, W\right) .
$$

Thus, by hypothesis,

$$
g\left(h_{2}(Z, W), X\right)=-X(\mu) g(Z, W),
$$

which shows that $M_{\perp}$ is totally umbilical in $M$. Moreover, by direct computations, we get

$$
\begin{aligned}
g\left(\nabla_{Z} \operatorname{grad} \mu, X\right) & =g\left(\nabla_{Z} \operatorname{grad} \mu, X\right) \\
& =\left[Z g(\operatorname{grad} \mu, X)-g\left(\operatorname{grad} \mu, \nabla_{Z} X\right)\right] \\
& =\left[Z(X(\mu))-[Z, X] \mu-g\left(\operatorname{grad} \mu, \nabla_{X} Z\right)\right] \\
& =\left[[Z, X] \mu+X(Z(\mu))-[Z, X] \mu-g\left(\operatorname{grad} \mu, \nabla_{X} Z\right)\right] \\
& =\left[X(Z(\mu))-g\left(\operatorname{grad} \mu, \nabla_{X} Z\right)\right] .
\end{aligned}
$$

Since $Z(\mu)=0$, we obtain

$$
g\left(\nabla_{Z} \operatorname{grad} \mu, X\right)=g\left(\operatorname{grad} \mu, \nabla_{X} Z\right) .
$$

On the other hand, since $\operatorname{grad} \mu \in \Gamma\left(T M_{\theta}\right)$ and $M_{\theta}$ is totally geodesic in $M$, it follows that $\nabla_{X} Z \in \Gamma\left(T M_{\perp}\right)$ for $X \in \Gamma\left(D^{\theta}\right)$ and $Z \in \Gamma\left(D^{\perp}\right)$. Hence $g\left(\nabla_{Z} \operatorname{grad} \mu, X\right)=0$. Then the spherical condition is also satisfied, that is, $M_{\perp}$ is an extrinsic sphere in $M$. Thus we conclude that $M$ is a warped product and the proof is complete.

REMARK 5.2. We note that the condition (5.2) in Theorem 5.1 is meaningless for CR-warped product submanifolds of Kaehler manifolds, because, from Lemma 4.1(5) of [7], it follows that any mixed geodesic CR-warped product submanifold is a CR-product. But that result is not true for mixed geodesic hemi-slant submanifolds. In the following we present an example of a mixed geodesic warped product hemi-slant submanifold which is not trivial.

EXAMPLE 5.2. Let $M$ be a submanifold of $\mathbb{R}^{8}$ given by

$$
\chi(u, v, \theta)=(u, v, \cos v, \sin v, 0, u \sin \theta, 0, u \cos \theta)
$$


for $\theta \in(0, \pi / 2), u \neq 0$, and $v \neq 0$. Then the tangent bundle $T M$ is spanned by

$$
\begin{aligned}
& Z_{1}=\frac{\partial}{\partial x_{1}}+\sin \theta \frac{\partial}{\partial x_{6}}+\cos \theta \frac{\partial}{\partial x_{8}}, \\
& Z_{2}=\frac{\partial}{\partial x_{2}}-\sin v \frac{\partial}{\partial x_{3}}+\cos v \frac{\partial}{\partial x_{4}}, \\
& Z_{3}=u \cos \theta \frac{\partial}{\partial x_{6}}-u \sin \theta \frac{\partial}{\partial x_{8}} .
\end{aligned}
$$

Then it is easy to see that $D^{\varphi}=\operatorname{span}\left\{Z_{1}, Z_{2}\right\}$ is a slant distribution with slant angle $\varphi=\pi / 3$. It is also easy to show that $D^{\perp}=\operatorname{span}\left\{Z_{3}\right\}$ is an anti-invariant distribution. Thus $M$ is a hemi-slant submanifold. Moreover, we can see that $D^{\varphi}$ and $D^{\perp}$ are integrable. Denote the integral manifolds of $D^{\varphi}$ and $D^{\perp}$ by $M_{\varphi}$ and $M_{\perp}$, respectively. Then the metric tensor of $M$ is

$$
g=2 d u^{2}+2 d v^{2}+u^{2} d \theta^{2} .
$$

Hence we have

$$
g=g_{M \varphi}+u^{2} g_{M_{\perp}} .
$$

Thus $M$ is a warped product hemi-slant submanifold of $\mathbb{R}^{8}$ with warping function $f=u$. On the other hand, the normal bundle $T M^{\perp}$ is spanned by

$$
\begin{array}{ll}
W_{1}=\sin \theta \frac{\partial}{\partial x_{5}}+\cos \theta \frac{\partial}{\partial x_{7}}, & W_{2}=-\frac{\partial}{\partial x_{1}}+\sin \theta \frac{\partial}{\partial x_{6}}+\cos \theta \frac{\partial}{\partial x_{8}}, \\
W_{3}=\cos v \frac{\partial}{\partial x_{2}}-\frac{\partial}{\partial x_{4}}, & W_{4}=\sin v \frac{\partial}{\partial x_{2}}+\frac{\partial}{\partial x_{3}}, \\
W_{5}=-u \cos \theta \frac{\partial}{\partial x_{5}}+u \sin \theta \frac{\partial}{\partial x_{7}} . &
\end{array}
$$

Then using the Gauss formula, we have

$$
h\left(Z_{1}, Z_{2}\right)=0, \quad h\left(Z_{1}, Z_{3}\right)=0, \quad h\left(Z_{2}, Z_{3}\right)=0, \quad h\left(Z_{1}, Z_{1}\right)=0
$$

and

$$
h\left(Z_{2}, Z_{2}\right)=\frac{\sin v}{1+\cos ^{2} v} W_{3}-\frac{\cos v}{1+\sin ^{2} v} W_{4}, \quad h\left(Z_{3}, Z_{3}\right)=-\frac{u}{2} W_{2} .
$$

Thus, $M$ is mixed geodesic, but it is neither totally geodesic nor totally umbilical.

LEMMA 5.2. Let $M$ be a warped product hemi-slant submanifold of a Kaehler manifold $\bar{M}$ of the form $M_{\theta} \times_{f} M_{\perp}$. Then

$$
g(h(X, Y), J Z)=g(h(X, Z), F Y)
$$

for $X, Y \in \Gamma\left(T M_{\theta}\right)$ and $Z \in \Gamma\left(T M_{\perp}\right)$.

Proof. From (2.2) we have $g(h(X, Y), J Z)=g\left(\bar{\nabla}_{X} Y, J Z\right)$. Then using $(2.1)$ we get $g(h(X, Y), J Z)=-g\left(\bar{\nabla}_{X} J Y, Z\right)$. Hence, $g(h(X, Y), J Z)=$ 
$g\left(J Y, \bar{\nabla}_{X} Z\right)$. Thus, from (2.5) we derive $g(h(X, Y), J Z)=g\left(T Y, \nabla_{X} Z\right)+$ $g(h(X, Z), F Y)$. Then (4.1) implies (5.3).

Let $M$ be an $(m+n)$-dimensional proper hemi-slant submanifold of a Kaehler manifold $\bar{M}^{m+n}$. Then we choose a canonical orthonormal frame $\left\{e_{1}, \ldots, e_{m}, \bar{e}_{1}, \ldots, \bar{e}_{n}, J e_{1}, \ldots, J e_{m}, e_{1}^{*}, \ldots, e_{n}^{*}\right\}$ of $\bar{M}$ such that, restricted to $M, e_{1}, \ldots, e_{m}, \bar{e}_{1}, \ldots, \bar{e}_{n}$ are tangent to $M$. Then $\left\{e_{1}, \ldots, e_{m}, \bar{e}_{1}, \ldots, \bar{e}_{n}\right\}$ form an orthonormal frame of $M$. We can take $\left\{e_{1}, \ldots, e_{m}, \bar{e}_{1}, \ldots, \bar{e}_{n}\right\}$ in such a way that $\left\{e_{1}, \ldots, e_{m}\right\}$ form an orthonormal frame of $D^{\perp}$ and $\left\{\bar{e}_{1}, \ldots, \bar{e}_{n}\right\}$ form an orhonormal frame of $D^{\theta}$, where $\operatorname{dim}\left(D^{\perp}\right)=m$ and $\operatorname{dim}\left(D^{\theta}\right)=n$. We can take $\left\{J e_{1}, \ldots, J e_{m}, e_{1}^{*}, \ldots, e_{n}^{*}\right\}$ in such a way that $\left\{J e_{1}, \ldots, J e_{n}\right\}$ form an orthonormal frame of $J\left(D^{\perp}\right)$ and $\left\{e_{1}^{*}, \ldots, e_{n}^{*}\right\}$ form an orthonormal frame of $F\left(D^{\theta}\right)$. It is known that a proper slant submanifold is always even-dimensional. Hence, $n=2 p$. Then we can choose orthonormal frames $\left\{\bar{e}_{1}, \ldots, \bar{e}_{2 p}\right\}$ of $D^{\theta}$ and $\left\{e_{1}^{*}, \ldots, e_{n}^{*}\right\}$ of $F\left(D^{\theta}\right)$ in such a way that

$$
\begin{aligned}
& \bar{e}_{1}=\sec \theta T \bar{e}_{1}, \ldots, \bar{e}_{2 p}=\sec \theta T \bar{e}_{2 p-1}, \\
& e_{1}^{*}=\csc \theta F \bar{e}_{1}, \ldots, e_{2 p}^{*}=\csc \theta F \bar{e}_{2 p},
\end{aligned}
$$

where $\theta$ is the slant angle. We note that such an orthonormal frame is called an adapted frame [6].

TheOREM 5.2. Let $M$ be an $(m+n)$-dimensional mixed geodesic warped product submanifold of the form $M_{\theta} \times_{f} M_{\perp}$ in a Kaehler manifold $\bar{M}^{m+n}$, where $M_{\theta}$ is a proper slant submanifold and $M_{\perp}$ is a totally real submanifold of $\bar{M}^{m+n}$. Then

(i) The squared norm of the second fundamental form of $M$ satisfies

$$
\|h\|^{2} \geq m \cot ^{2} \theta\|\nabla(\ln f)\|^{2}, \quad \operatorname{dim}\left(M_{\perp}\right)=m .
$$

(ii) If equality holds identically in (5.4), then $M_{\theta}$ is a totally geodesic submanifold and $M_{2}$ is a totally umbilical submanifold of $\bar{M}$. Moreover, $M$ is never a minimal submanifold of $\bar{M}$.

Proof. Since

$$
\|h\|^{2}=\left\|h\left(D^{\theta}, D^{\theta}\right)\right\|^{2}+\left\|h\left(D^{\perp}, D^{\perp}\right)\right\|^{2}+2\left\|h\left(D^{\theta}, D^{\perp}\right)\right\|^{2},
$$

if $M$ is mixed geodesic we have

$$
\|h\|^{2}=\sum_{k=1}^{m+2 p} \sum_{i, j=1}^{m} g\left(h\left(e_{i}, e_{j}\right), \tilde{e}_{k}\right)^{2}+\sum_{k=1}^{m+2 p} \sum_{r, s=1}^{2 p} g\left(h\left(\bar{e}_{r}, \bar{e}_{s}\right), \tilde{e}_{k}\right)^{2},
$$

where $\left\{\tilde{e}_{k}\right\}$ is an orthonormal basis of $T M^{\perp}$. Now, considering the adapted 
frame, we can rewrite the above equation as

$$
\begin{aligned}
\|h\|^{2}= & \sum_{l=1}^{m} \sum_{i, j=1}^{m} g\left(h\left(e_{i}, e_{j}\right), J e_{l}\right)^{2}+\sum_{a=1}^{2 p} \sum_{i, j=1}^{m} g\left(h\left(e_{i}, e_{j}\right), \csc \theta F \bar{e}_{a}\right)^{2} \\
& +\sum_{l=1}^{m} \sum_{r, s=1}^{2 p} g\left(h\left(\bar{e}_{r}, \bar{e}_{s}\right), J e_{l}\right)^{2}+\sum_{a=1}^{2 p} \sum_{r, s=1}^{2 p} g\left(h\left(\bar{e}_{r}, \bar{e}_{s}\right), \csc \theta F \bar{e}_{a}\right)^{2} .
\end{aligned}
$$

Then, from (5.1) and (5.3), we obtain

$$
\begin{aligned}
\|h\|^{2}= & \sum_{l=1}^{m} \sum_{i, j=1}^{m} g\left(h\left(e_{i}, e_{j}\right), J e_{l}\right)^{2}+\sum_{a=1}^{2 p} \sum_{i, j=1}^{m} \csc ^{2} \theta\left(T \bar{e}_{a}(\ln f)\right)^{2} g\left(e_{i}, e_{j}\right)^{2} \\
& +\sum_{a=1}^{2 p} \sum_{r, s=1}^{2 p} g\left(h\left(\bar{e}_{r}, \bar{e}_{s}\right), \csc \theta F \bar{e}_{a}\right)^{2} .
\end{aligned}
$$

Hence,

$$
\begin{aligned}
\|h\|^{2}= & \sum_{l=1}^{m} \sum_{i, j=1}^{m} g\left(h\left(e_{i}, e_{j}\right), J e_{l}\right)^{2}+m \sum_{a=1}^{2 p} \csc ^{2} \theta\left(T \bar{e}_{a}(\ln f)\right)^{2} \\
& +\sum_{a=1}^{2 p} \sum_{r, s=1}^{2 p} g\left(h\left(\bar{e}_{r}, \bar{e}_{s}\right), \csc \theta F \bar{e}_{a}\right)^{2} .
\end{aligned}
$$

On the other hand, by direct computations, using adapted frame, we get

$$
\begin{aligned}
\sum_{a=1}^{2 p} \csc ^{2} \theta\left(T \bar{e}_{a}(\right. & \ln f))^{2} \\
=[ & {\left[\bar{e}_{1}(\ln f)\right]^{2} \csc ^{2} \theta+\left[\sec \theta T^{2} \bar{e}_{1}(\ln f)\right]^{2} \csc ^{2} \theta } \\
& +\left[T \bar{e}_{2}(\ln f)\right]^{2} \csc ^{2} \theta+\left[\sec \theta T^{2} \bar{e}_{2}(\ln f)\right]^{2} \csc ^{2} \theta+\cdots \\
& +\left[T \bar{e}_{2 p-1}(\ln f)\right]^{2} \csc ^{2} \theta+\left[\sec \theta T^{2} \bar{e}_{2 p-1}(\ln f)\right]^{2} \csc ^{2} \theta
\end{aligned}
$$

Then, rearranging this equation and using Theorem 3.2, we arrive at

$$
\begin{aligned}
\sum_{a=1}^{2 p} \csc ^{2} \theta\left(T \bar{e}_{a}(\ln f)\right)^{2} \\
=\left[\sec \theta T \bar{e}_{1}(\ln f)\right]^{2} \cos ^{2} \theta \csc ^{2} \theta+\left[\cos \theta \bar{e}_{1}(\ln f)\right]^{2} \csc ^{2} \theta \\
\quad+\left[\sec \theta T \bar{e}_{2}(\ln f)\right]^{2} \cos ^{2} \theta \csc ^{2} \theta+\left[\cos \theta \bar{e}_{2}(\ln f)\right]^{2} \csc ^{2} \theta+\cdots \\
\quad+\left[\sec \theta T \bar{e}_{2 p-1}(\ln f)\right]^{2} \cos ^{2} \theta \csc ^{2} \theta+\left[\cos \theta \bar{e}_{2 p-1}(\ln f)\right]^{2} \csc ^{2} \theta
\end{aligned}
$$


Hence we get

$$
\sum_{a=1}^{2 p} \csc ^{2} \theta\left(T \bar{e}_{a}(\ln f)\right)^{2}=\cot ^{2} \theta\|\nabla(\ln f)\|^{2} .
$$

Thus using (5.6) in (5.5) we obtain the inequality (5.4). If equality holds in (5.4), we have

$$
\sum_{l=1}^{m} \sum_{i, j=1}^{m} g\left(h\left(e_{i}, e_{j}\right), J e_{l}\right)^{2}=0, \quad \sum_{a=1}^{2 p} \sum_{r, s=1}^{2 p} g\left(h\left(\bar{e}_{r}, \bar{e}_{s}\right), \csc \theta F \bar{e}_{a}\right)^{2}=0 .
$$

Since $M$ is mixed geodesic, from (5.3) we also have

$$
g(h(X, Y), J Z)=0, \quad \forall X, Y \in \Gamma\left(T M_{\theta}\right), Z \in \Gamma\left(T M_{\perp}\right) .
$$

Since $M_{\theta}$ is totally geodesic in $M$, the second condition of (5.7) and (5.8) imply that $M_{\theta}$ is totally geodesic in $\bar{M}$. On the other hand, the first condition of (5.7) implies

$$
h(Z, W) \in \Gamma\left(F\left(D^{\theta}\right)\right) .
$$

From (5.1) we get

$$
g(h(Z, W), F X)=T X(\ln f) g(Z, W)
$$

for $Z, W \in \Gamma\left(T_{\perp}\right)$ and $X \in \Gamma\left(T M_{\theta}\right)$. These equations imply that $M_{\perp}$ is totally umbilical in $\bar{M}$. Moreover, from (5.9) and (5.6) we conclude that if $M$ is minimal, then $\|\nabla(\ln f)\|^{2}=0$, which is a contradiction.

REMARK 5.3. It is well known that the semi-slant submanifolds were introduced as a generalization of proper slant and proper CR-submanifolds. From Theorem 4.1, it follows that the semi-slant submanifolds in the sense of N. Papaghiuc are not useful to generalize the CR-warped products. But, from Remark 5.1 and Examples 5.1-5.2, one can conclude that warped product hemi-slant submanifolds of the form $M_{\theta} \times_{f} M_{\perp}$ are a generalization of CR-warped products in Kaehler manifolds.

\section{References}

[1] A. Bejancu, Geometry of CR-Submanifolds, Kluwer, Dordrecht, 1986.

[2] R. L. Bishop and B. O'Neill, Manifolds of negative curvature, Trans. Amer. Math. Soc. 145 (1969), 1-49.

[3] V. Bonanzinga and K. Matsumoto, Warped product CR-submanifolds in locally conformal Kaehler manifold, Period. Math. Hungar. 48 (2004), 207-221.

[4] J. L. Cabrerizo, A. Carriazo, L. M. Fernández and M. Fernández, Semi-slant submanifolds of a Sasakian manifold, Geom. Dedicata 78 (1999), 183-199.

[5] A. Carriazo, Bi-slant immersions, in: Proc. ICRAMS 2000, Kharagpur, India, 2000, 88-97.

[6] B. Y. Chen, Geometry of Slant Submanifolds, Katholieke Universiteit Leuven, Leuven, 1990. 
B. Sahin

[7] B. Y. Chen, Geometry of warped product CR-submanifolds in Kaehler manifolds, Monatsh. Math. 133 (2001), 177-195.

[8] -, Geometry of warped product CR-submanifolds in Kaehler manifolds II, ibid. 134 (2001), 103-119.

[9] - CR-warped products in complex projective spaces with compact holomorphic factor, ibid. 141 (2004), 177-186.

[10] F. Dillen and S. Nölker, Semi-parallelity, multi-rotation surfaces and the helixproperty, J. Reine Angew. Math. 435 (1993), 33-63.

[11] I. Hasegawa and I. Mihai, Contact CR-warped product submanifolds in Sasakian manifolds, Geom. Dedicata 102 (2003), 143-150.

[12] S. Hiepko, Eine innere Kennzeichnung der verzerrten Produkte, Math. Ann. 241 (1979), 209-215.

[13] I. Mihai, Contact CR-warped product submanifolds in Sasakian space forms, Geom. Dedicata 109 (2004), 165-173.

[14] M. I. Munteanu, Warped product contact CR-submanifolds of Sasakian space forms, Publ. Math. Debrecen 66 (2005), 75-120.

[15] —, Doubly warped product CR-submanifolds in locally conformal Kähler manifolds, Monatsh. Math. 150 (2007), 333-342.

[16] N. Papaghiuc, Semi-slant submanifolds of a Kaehlerian manifold, An. Ştiinţ. Al. I. Cuza Univ. Iaşi 40 (1994), 55-61.

[17] B. Sahin, Non-existence of warped product semi-slant submanifolds of Kaehler manifolds, Geom. Dedicata 117 (2006), 195-202.

[18] K. Yano and M. Kon, CR-submanifolds of Kaehlerian and Sasakian Manifolds, Birkhäuser, 1983.

Department of Mathematics

Inonu University

44280, Malatya, Turkey

E-mail: bsahin@inonu.edu.tr

Received 15.5.2008

and in final form 2.2.2009 